\title{
High resistance against clindamycin, metronidazole and amoxicillin in Porphyromonas gingivalis and Aggregatibacter actinomycetemcomitans isolates of periodontal disease
}

\author{
Carlos M. Ardila ${ }^{1,2}$, Mayra A. López ${ }^{2}$, Isabel C. Guzmán ${ }^{2}$ \\ ${ }^{1}$ Periodontist Epidemiology Group, University of Antioquia, Colombia \\ ${ }^{2}$ Periodontist Dentist Department of Periodontology, School of Dentistry, University of Antioquia, Colombia
}

Correspondence:

Calle $64 N^{\circ} 52-59$

Medellín, Colombia

martinardila@gmail.com

Received: 17/09/2009

Accepted: 21/02/2010

\author{
Ardila CM, López MA, Guzmán IC. High resistance against clindamy- \\ cin, metronidazole and amoxicillin in Porphyromonas gingivalis and $\mathrm{Ag}$ - \\ gregatibacter actinomycetemcomitans isolates of periodontal disease. \\ Med Oral Patol Oral Cir Bucal. 2010 Nov 1;15 (6):e947-51. \\ http://www.medicinaoral.com/medoralfree01/v15i6/medoralv15i6p947.pdf

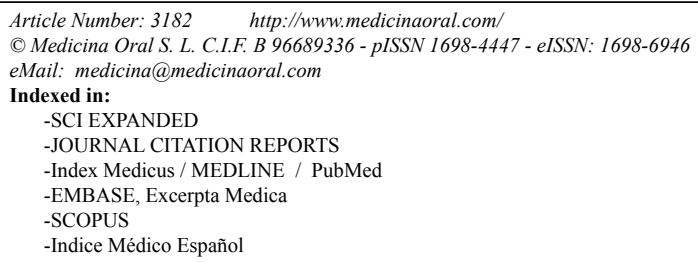

\begin{abstract}
Objectives: To test the antimicrobial sensitivity of two periodontal pathogens to a panel of five orally administrable antibiotics in periodontal disease.

Study design: A total of 69 isolates of Aggregatibacter actinomycetemcomitans and Porphyromonas gingivalis were processed using culture and biochemical tests. Selected colonies of A. actinomycetemcomitans and P. gingivalis were used to evaluate the antibacterial activity of clindamycin, metronidazole, amoxicillin, moxifloxacin and amoxicillin/clavulanic acid.

Results: Susceptibility testing revealed a sensitivity of $100 \%$ of $A$. actinomycetemcomitans and $P$. gingivalis to moxifloxacin and amoxicillin/clavulanic acid but moderate susceptibilities were found for the rest of antibiotics agents evaluated.

Conclusions: The widespread use of antibiotics is reflected in the level of resistance of A. actinomycetemcomitans and $P$. gingivalis in patients with periodontal infections. This suggests that antibiotic susceptibility testing is necessary to determine efficacy of antimicrobial agents. Clinical studies with antibiotics should take these differences into account.
\end{abstract}

Key words: Antibiotics, antimicrobial activity, resistance.

\section{Introduction}

Periodontitis, a chronic inflammatory disease, begins with a microbial infection, followed by a host-mediated destruction of soft tissue caused by hyperactivated or primed leukocytes and the generation of cytokines, eicosanoids, and matrix metalloproteinases that cause clinically significant connective tissue and bone de- struction (1). Aggregatibacter actinomycetemcomitans and Porphyromonas gingivalis are implicated in the pathogenesis of periodontitis $(2,3)$. These bacteria are able to produce virulence factors that act locally within the sulcus, and result in tissue destruction $(2,3)$. Examples of virulence factors include proteolytic enzymes produced by $P$. gingivalis and leukotoxins produced by A. actinomycetemcomitans (3). P. gingivalis has shown 
the ability to invade human gingival fibroblasts in cell culture while $P$. gingivalis and $A$. actinomycetemcomitans have all demonstrated the ability to invade human oral epithelial cells in cell culture (4). By the way, it is noteworthy that $35 \%$ of nosocomial pneumonia has been demonstrated to be related to periodontopathogens (5). Invasiveness and ubiquitous intraoral distribution may be the main reasons for the reported observation of rather poor results after conventional, merely mechanical treatment of periodontal infections (6). Thus, adjunctive systemic antibiotics have been advocated in order to suppress the organism in subgingival plaque (7). The adjunctive use of systemically administered antibiotics has been shown to provide a better clinical outcome, particularly in terms of pocket depth reduction and attachment-level gain, than scaling and root planing in chronic periodontitis subjects $(6,7)$. Commonly used antibiotics include clindamycin, metronidazole and amoxicillin $(6,8)$. Besides, positive responses have been reported with amoxicillin/clavulanic $(6,8)$ and moxifloxacin (7) in the treatment of periodontitis.

Most of the knowledge available on the microbial composition of subgingival plaque is based on studies from the United States and Europe (8-10), where therapeutic oral care programs are available to significant portions of the population. Temporal and geographic changes in antibiotic susceptibility among anaerobes have been reported in Europe $(8,10)$. Besides, the development of resistance and significant variation in the local prevalence of resistance are well recognized in aerobic and anaerobic bacteria in Latin America (9). Determinations of MIC values permit early recognition of emerging resistance. For relevant comparative analysis distributions of the minimal inhibitory concentration (MIC) values are mandatory as the terms susceptible, intermediate and resistant are not universally applicable since breakpoints may vary in different countries (11).

To the best of our knowledge, there are no studies that determine the resistance of $A$. actinomycetemcomitans and $P$. gingivalis to a panel of five orally administrable antibiotics in periodontal disease. Thus, the present study aimed to test, in vitro, the susceptibility to different antibiotics of $A$. actinomycetemcomitans and $P$. gingivalis isolated from subgingival plaque in periodontal infections.

\section{Study Design}

Population studied, clinical evaluation, microbiological sampling and microbiological procedures.

A total of 76 adult patients in good general health and with no current medication usage were included in the study after giving informed consent. Medical history and clinical and radiographic examination were conducted for each patient. One of the authors (CA) carried out a complete periodontal examination. The following clinical parameters were recorded: probing depth (PD), clinical attachment level (CAL), and percentages of sites with bleeding on probing (BOP) and plaque. A marked probe (UNC-15, Hu-Friedy, Chicago, IL) was used in all instances. Periodontal diagnosis was established based on the consensus report of the American Academy of Periodontology (AAP) (12).

Subgingival microbial samples were taken from the six deepest pockets. After removing supragingival plaque with curets and isolating the area with cotton pellets, the paper points were inserted into each periodontal pocket for 20 seconds. The paper points (Maillefer, Ballaigues, Switzerland) were pooled in screw cap vials containing Viability Medium Götenborg Anaerobically (VMGA) III medium (13). All samples were labeled properly and processed within 4 hours after sampling. The samples were analyzed using microbial culture techniques for the presence of periodontopathic bacteria according to Slots (14). Briefly, most samples were processed at room temperature $\left(25^{\circ} \mathrm{C}\right)$ and incubated in $\mathrm{CO}_{2}$ and anaerobic culture systems. Brucella blood agar medium was incubated at $35^{\circ} \mathrm{C}$ in an anaerobic jar for 7 days. The Trypticicase Soy Serum Bacitracin Vancomycin agar (TSBV) medium was incubated in $10 \% \mathrm{CO}_{2}$ at $37^{\circ} \mathrm{C}$ for 4 days. Presumptive identification was performed according to the methods described (14), and using a commercial identification micromethod system (RapID ANA II, Remel, Norcross, GA, USA) for A. actinomycetemcomitans and $P$. gingivalis. Total viable counts (TVC) were defined as the total number of colonyforming units obtained on non-selective media plates. Species found on selective media were enumerated and their percentage of TVC was calculated.

\section{Antimicrobial susceptibility testing}

The susceptibility/resistance of selected colonies of $A$. actinomycetemcomitans and $P$. gingivalis from pure cultures to five antibiotics were measured. The following antimicrobials were tested: clindamycin, metronidazole, amoxicillin, moxifloxacin and amoxicillin/ clavulanic acid (E-test ${ }^{\circledR}$, AB Biodisk, Solna, Sweden). Briefly, viable colonies were homogenized in $0.85 \%$ saline, and the turbidity was adjusted to MacFarland 1.0 standard (3 x $108 \mathrm{CFU} / \mathrm{ml})$. Using a sterile glass rod, $0.1 \mathrm{ml}$ of the inoculums was spread over Brucella blood agar plates (BD, Sparks, MD, USA) and dried for 15 minutes at room temperature. E-test strips were gently placed onto the agar surface and incubated under anaerobic conditions for 4 days. The elliptic zone of inhibition was examined after 96 hours of incubation. The reading at the intersection of the bacterial zone of inhibition and the E-strip represented the MIC of the organism. The MIC breakpoints were interpreted according to the Clinical Laboratory Standards Institute guidelines (15). In the case of $A$. actinomycetemcomitans, the interpretive criteria for the HACEK group were applied 
for amoxicillin/clavulanic acid, whereas for metronidazole those for anaerobes were used. As no interpretive criteria exist for clindamycin, the interpretive criteria for anaerobes were applied (15).

Statistical Analysis

Descriptive analyses were carried out for clinical and microbiological parameters. A statistical program was used for all the statistical analyses (SPSS, Statistical Package for the Social Sciences, version 15, Chicago, IL).

\section{Results}

Table 1 depicts the clinical characteristics of study subjects. A total of 45 women (59.2\%) and 31 men (40.8\%) with chronic periodontitis were studied. From the 76 study patients a total of 69 bacterial colonies could be

Table 1. Clinical parameters of the periodontal sites being selected for subgingival plaque sampling.

\begin{tabular}{|l|l|}
\hline Clinical Parameter & Mean SD \\
\hline Clinical attachment level $(\mathrm{mm} \pm \mathrm{SD})$ & $4.3 \pm 1.99$ \\
\hline Probing depth $(\mathrm{mm} \pm \mathrm{SD})$ & $3.4 \pm 1.48$ \\
\hline$\%$ sites with plaque $(\mathrm{mm} \pm \mathrm{SD})$ & $56 \pm 28$ \\
\hline$\%$ of sites with bleeding on probing $(\mathrm{mm} \pm \mathrm{SD})$ & $77 \pm 21$ \\
\hline
\end{tabular}

clearly identified. Table 2 shows the in vitro susceptibility of $A$. actinomycetemcomitans $(\mathrm{n}=18)$ and $P$. gingivalis $(\mathrm{n}=51)$ to the five antibiotics tested. Susceptibility testing revealed a sensitivity of $100 \%$ of A. actinomycetemcomitans and $P$. gingivalis to moxifloxacin and amoxicillin/clavulanic acid but moderate susceptibilities were found for the rest of antibiotics agents evaluated.

\section{Discussion}

The overuse, misuse and widespread prophylactic application of antimicrobial drugs are some of the factors that have led to the emergence of drug resistant microorganisms $(11,16)$. Microbial resistance has become a worldwide medical, economic and public health problem although microbial resistance rates are not equally distributed around the world $(9-11,16)$. A. actinomycetemcomitans and $P$. gingivalis represent two important periodontal pathogens. In this study, we investigated the susceptibility of $P$. gingivalis and A. actinomycetemcomitans from patients with untreated chronic periodontitis; their invasiveness and ubiquitous intraoral distribution may be the main reasons for the reported observation of poor results after mechanical therapy (6). Additionally, bacterial resistance to penicillin has become a problem of great clinical significance in Latin America because of its widespread use for many years $(11,16)$.

In our study, $P$. gingivalis was highly susceptible to moxifloxacin and amoxicillin/clavulanic acid (Table 2). These findings are in agreement with previous studies which have found that $P$. gingivalis is highly susceptible to these antibiotics $(7,8,17,18)$. Nevertheless, in our study $23.52 \%, 21.56 \%$ and $25.49 \%$ of the isolates were

Table 2. Minimal inhibitory concentration (MIC, $\mathrm{mg} / \mathrm{ml}$ ) across 18 strains of A. actinomycetemcomitans and 51 strains of $P$. gingivalis.

\begin{tabular}{|l|c|c|c|c|c|c|}
\hline & & $\mathbf{M I C}$ & $\mathbf{( m g / L})^{\mathbf{a}}$ & & & \\
\hline Microorganism and antibiotic & Range & $50 \%$ & $90 \%$ & $\mathrm{R}^{\mathrm{b}}(\%)$ & $\mathrm{IR}^{\mathrm{c}}(\%)$ & $\mathrm{S}^{\mathrm{d}}(\%)$ \\
\hline & & & & & & \\
A. actinomycetemcomitans & & & & & & \\
Clindamycin & $0.016>256$ & 0.125 & $>256$ & 83.33 & 5.56 & 11.11 \\
Metronidazole & $1.5>256$ & 6 & $>256$ & 88.88 & 5.56 & 5.56 \\
Amoxicillin & $0.064-32$ & 0.25 & 32 & 77.77 & - & 22.23 \\
Moxifloxacin & $0.19-0.5$ & 0.38 & 0.5 & - & - & 100 \\
Amoxicillin/clavulanic acid & $0.02-0.75$ & 0.25 & 0.5 & - & - & 100 \\
& & & & & & \\
\hline & & & & & & \\
P. gingivalis & & & & & & \\
Clindamycin & $0.08 \geq 16$ & 8 & $\geq 16$ & 23.52 & 7.8 & 68.68 \\
Metronidazole & $0.08 \geq 16$ & 0.256 & $\geq 16$ & 21.56 & 9.8 & 68.64 \\
Amoxicillin & $0.016>256$ & 0.125 & $>256$ & 25.49 & - & 74.51 \\
Moxifloxacin & $0.006-0.032$ & 0.023 & 0.032 & - & - & 100 \\
Amoxicillin/clavulanic acid & $<0.016-0.064$ & $<0.016$ & $<0.016$ & - & - & 100 \\
& & & & & & \\
\hline
\end{tabular}

a 50\% and $90 \%$ indicate the MIC values at which $50 \%$ and $90 \%$ of isolates were inhibited respectively. bResistance. cIntermediate resistance. dSensitive 
non-susceptible to clindamycin, metronidazole and amoxicillin respectively. Similar results have been reported by other investigations that detected resistances, although lower (19). In this viewpoint, it is important to note that $P$. gingivalis has recently been shown to be able of transfer plasmid DNA, chromosomal DNA, or both which would supply a functional system to also transfer resistance determinants (2).

The present findings support that A. actinomycetemcomitans was the least vulnerable microorganisms. Amongst the drugs tested, moxifloxacin and amoxicillin/clavulanic were active against all $A$. actinomycetemcomitans isolates in vitro (Table 2). Previous studies of the susceptibility of $A$. actinomycetemcomitans have also shown high susceptibility to these two antibiotics $(6-8,17,18)$. In this study, clindamycin, metronidazole and amoxicillin resistance was found amongst $A$. actinomycetemcomitans isolates. Clindamycin, metronidazole and amoxicillin resistance observed in this study agrees with the studies of van Winkelhoff et al. (8), Madinier et al. (20), and Kulik et al. (17) where $82 \%, 72 \%$ and $33 \%$ of the A. actinomycetemcomitans isolates were nonsusceptible. Once again resistance values found in our study were higher. On the other hand, metronidazole in combination with amoxicillin has been shown to be successful in the treatment of $A$. actinomycetemcomitans associated periodontal disease (6). In contrast, the MIC90 value of $>256 \mu \mathrm{g} / \mathrm{ml}$ of the Spanish A. actinomycetemcomitans may indicate that the clinical value of this mixture therapy may not be equal in Spain as it is in other European countries (8). Analogous situations may possibly arise in Latin America.

Multidrug-resistance is of great concern in skin and soft tissue infections, especially in Latin America with a calculated occurrence rate of $24.7 \%$ compared with markedly lower rates in Europe (10.8\%) and North America (3.2\%) (16). Latin America also has the lowest susceptibilities of all regions for the following antimicrobials: aztreonam, cefepime, ceftazidime, gentamicin, ciprofloxacin, imipenem, polymyxin B, and piperacillin/ tazobactam (16). Imipenem susceptibilities were lowest in Latin America at $65.3 \%$ compared with Europe $(80.7 \%)$ and North America (88.7\%) (16). Imipenem resistance is due in part to the appearance and dissemination of metallo- $\beta$-lactamase enzymes that have been described repeatedly in Latin America (16). Moreover, an increasing prevalence of penicillin resistant pneumococci has been observed throughout the world, and Latin America echoes this trend (11).

The results of the present study have shown that moxifloxacin and amoxicillin/clavulanic acid were the most active antibiotics against $A$. actinomycetemcomitans and $P$. gingivalis with all the isolates being susceptible. Less effective were clindamycin, metronidazole and amoxicillin. Owing to geographical differences as well as differences over time, we suggest that the overuse and misuse of antibiotics could be influencing the manifestation of more highly resistant strains associated with periodontal infections in our population. Ongoing longitudinal surveillance studies have been crucial to the detection and monitoring of regional antimicrobial resistance patterns, and continue to provide important insights that may serve to modify local prescribing guidelines.

\section{References}

1. Friedewald VE, Kornman KS, Beck JD, Genco R, Goldfine A, Libby P, et al. The American Journal of Cardiology and Journal of Periodontology editors' consensus: periodontitis and atherosclerotic cardiovascular disease. J Periodontol. 2009;80:1021-32.

2. Tribble GD, Lamont GJ, Progulske-Fox A, Lamont RJ. Conjugal transfer of chromosomal DNA contributes to genetic variation in the oral pathogen Porphyromonas gingivalis. J Bacteriol. 2007; 189:6382-8.

3. Fives-Taylor PM, Meyer DH, Mintz KP, Brissette C. Virulence factors of Actinobacillus actinomycetemcomitans. Periodontol 2000. 1999;20:136-67.

4. Rudney JD, Chen R, Sedgewick GJ. Intracellular Actinobacillus actinomycetemcomitans and Porphyromonas gingivalis in buccal epithelial cells collected from human subjects. Infect Immun. 2001;69:2700-7.

5. Bartlett JG, O’Keefe P, Tally FP, Louie TJ, Gorbach SL. Bacteriology of hospital-acquired pneumonia. Arch Intern Med. 1986;146:86871.

6. Walker C, Karpinia K. Rationale for use of antibiotics in periodontics. J Periodontol. 2002;73:1188-96.

7. Guentsch A, Jentsch H, Pfister W, Hoffmann T, Eick S. Moxifloxacin as an adjunctive antibiotic in the treatment of severe chronic periodontitis. J Periodontol. 2008;79:1894-903.

8. Van Winkelhoff AJ, Herrera D, Oteo A, Sanz M. Antimicrobial profiles of periodontal pathogens isolated from periodontitis patients in The Netherlands and Spain. J Clin Periodontol. 2005;32:893-8.

9. Herrera D, Contreras A, Gamonal J, Oteo A, Jaramillo A, Silva N, et al. Subgingival microbial profiles in chronic periodontitis patients from Chile, Colombia and Spain. J Clin Periodontol. 2008;35:10613.

10. Mosca A, Miragliotta L, Iodice MA, Abbinante A, Miragliotta G. Antimicrobial profiles of Prevotella spp. and Fusobacterium nucleatum isolated from periodontal infections in a selected area of southern Italy. Int J Antimicrob Agents. 2007;30:521-4.

11. Valenzuela MT, de Quadros C. Antibiotic resistance in Latin America: a cause for alarm. Vaccine. 2009;27 Suppl 3:C25-8.

12. Armitage GC. Development of a classification system for periodontal diseases and conditions. Ann Periodontol. 1999;4:1-6.

13. Möller AJ. Microbiological examination of root canals and periapical tissues of human teeth. Methodological studies. Odontol Tidskr. 1966;74:1-380.

14. Slots J. Rapid identification of important periodontal microorganisms by cultivation. Oral Microbiol Immunol. 1986;1:48-57.

15. Ambler J, Rennie R, Poupard J, Koeth L, Stass H, Endermann R, et al. Determination of moxifloxacin anaerobic susceptibility breakpoints according to the Clinical and Laboratory Standards Institute guidelines. Diagn Microbiol Infect Dis. 2008;61:49-57.

16. Moet GJ, Jones RN, Biedenbach DJ, Stilwell MG, Fritsche TR. Contemporary causes of skin and soft tissue infections in North America, Latin America, and Europe: report from the SENTRY Antimicrobial Surveillance Program (1998-2004). Diagn Microbiol Infect Dis. 2007;57:7-13.

17. Kulik EM, Lenkeit K, Chenaux S, Meyer J. Antimicrobial susceptibility of periodontopathogenic bacteria. J Antimicrob Chemother. 2008;61:1087-91. 
18. Brescó-Salinas M, Costa-Riu N, Berini-Aytés L, Gay-Escoda C. Antibiotic susceptibility of the bacteria causing odontogenic infections. Med Oral Patol Oral Cir Bucal. 2006;11:E70-5.

19. Eick S, Pfister W, Straube E. Antimicrobial susceptibility of anaerobic and capnophilic bacteria isolated from odontogenic abscesses and rapidly progressive periodontitis. Int $\mathrm{J}$ Antimicrob Agents. 1999;12:41-6.

20. Madinier IM, Fosse TB, Hitzig C, Charbit Y, Hannoun LR. Resistance profile survey of 50 periodontal strains of Actinobacillus actinomyectomcomitans. J Periodontol. 1999;70:888-92.

\section{Conflict of Interest and Sources of Funding Statement}

This study was supported by a grant from the National Public Health School and Epidemiology Group of the University of Antioquia.

The authors declare that there are no conflicts of interest in this study. 